\title{
DIVERTÍCULO ESOFÁGICO FISTULADO EM UM EQUINO DA RAÇA CRIOULA - RELATO DE CASO
}

\author{
MONTELLO NETO, Joel de Souza ${ }^{1}$; \\ MORAES, Angélica Trazzi Bento de ${ }^{2}$; \\ CORAUCCI, Maria Virgínia de Oliveira ${ }^{3}$; \\ DANEZE, Edmilson Rodrigo ${ }^{3}$; \\ DIAS, Bianca Paludeto ${ }^{1}$; \\ HARAGUCHI, Greice Daieni Akemi ${ }^{4}$; \\ PAULA, Regiane Martins de ${ }^{4}$.
}

\footnotetext{
${ }^{1}$ Médico Veterinário, Hospital Veterinário da Faculdade Dr. Francisco Maeda/FAFRAM/FE, Ituverava-SP;

${ }^{2}$ Médica Veterinária, Doutora, Professora do curso de Medicina Veterinária da Faculdade Dr. Francisco Maeda/FAFRAM/FE, Ituverava-SP; ${ }^{3}$ Médico Veterinário, Programa de Aprimoramento Profissional em Clínica e Cirurgia de Grandes Animais da Faculdade Dr. Francisco Maeda/FAFRAM/FE, Ituverava-SP; ${ }^{4}$ Médica Veterinária,

Programa de Aprimoramento Profissional em Clínica e Cirurgia Veterinária da Faculdade Dr. Francisco Maeda/FAFRAM/FE, Ituverava-SP.
}

\section{RESUMO}

$\mathrm{D}$ ivertículos esofágicos são dilatações focais da camada muscular do esôfago, podendo ser congênitos ou adquiridos. O presente trabalho tem como objetivo relatar o caso de um equino da raça Crioula que apresentava aumento de volume cervical de consistência firme, na posição ventrolateral, com discreto deslocamento à esquerda, disfagia e dispneia transitória. No exame radiográfico visualizou-se uma massa de aspecto oval com conteúdo radiopaco, entre $\mathrm{C} 2-\mathrm{C} 4$, característico de divertículo esofágico. Durante o procedimento cirúrgico, observou-se exuberante aderência da musculatura cervical e artéria carótida esquerda com a massa responsável pelo aumento de volume na região. Isolada a massa, a mesma foi seccionada e identificada como sendo um abscesso decorrente de uma fístula no divertículo, optando-se pela realização de uma diverticulectomia modificada. Através desse caso, concluímos que a ocorrência de um divertículo esofágico fistulado, com formação subsequente de abscesso e aderido a estruturas importantes, dificulta o tratamento cirúrgico, sendo necessária uma análise individual e, eventualmente, um procedimento cirúrgico modificado para a sua correção.

Palavras-chave: Abscesso. Alteração anatômica. Disfagia. Dispneia. Esôfago. 


\section{INTRODUÇÃO}

Em equinos, citam-se como afeç̧ões esofágicas as obstruções, estenoses, rupturas, megaesôfago (STICK, 1990), fístulas ou neoplasias (VRINS et al., 1983). No entanto, raramente são descritos casos de ectasia, arco aórtico persistente, cistos esofágicos (MURRAY, 2000) ou divertículos (MURRAY; GAUGHAN, 1993) na espécie.

Divertículos esofágicos são dilatações focais da camada muscular do esôfago, podendo ser adquiridos (decorrentes de traumas, lesões cervicais ou corpos estranhos) ou congênitos (HIGGINS; SNYDER, 2006; STICK, 2011; VRINS et al., 1983). Os divertículos adquiridos são os mais comuns na espécie equina, sendo classificados por tração (verdadeiro) ou pulsão (falso) (FUBINI, 2002; MURRAY, 2000; STICK, 1990).

Os sinais clínicos são os mesmos de um animal apresentando obstrução esofágica, em que se observam principalmente disfagia, tosse durante a deglutição, regurgitação nasal e sialorreia (MACORIS et al., 2008; MURRAY; GAUGHAN, 1993); dependendo do grau de obstrução, observa-se aumento de volume cervical (MACORIS et al., 2008; STICK, 1990), além de perda de peso progressiva, desidratação e desequilíbrio eletrolítico (STICK, 1999; STICK, 2011). Na maioria dos casos existe uma dificuldade de passagem da sonda nasogástrica, ressaltando-se a possibilidade de ocorrerem rupturas e o consequente acúmulo de conteúdo esofágico na região cervical e/ou torácica (STICK, 2011; VRINS et al., 1983); por isso o esôfago deve ser avaliado minuciosamente, sendo de suma importância a utilização de radiografias contrastadas para a conclusão do diagnóstico (BLICKSLAGER; JONES, 2006) e realização do tratamento adequado (STICK, 2011; VRINS et al., 1983). O prognóstico depende do tipo, tamanho e persistência da afecção (HIGGINS; SNYDER, 2006).

São de extrema importância relatos de alterações anatômicas em equinos, pois a medicina veterinária evolui a cada dia, proporcionando melhorias nos tratamentos e nos diagnósticos das afecções, auxiliando assim, na qualidade de vida dos animais. Nesse contexto, o presente trabalho teve como objetivo relatar a ocorrência de um caso de divertículo esofágico fistulado em um equino da raça Crioula, enfatizando o diagnóstico e o tratamento utilizados. 


\section{RELATO DE CASO}

Um equino, fêmea, da raça Crioula, de 8 anos de idade, pesando $400 \mathrm{~kg}$, foi encaminhado para o Hospital Veterinário da Faculdade Dr. Francisco Maeda (FAFRAM/FE), localizado em Ituverava-SP, com histórico de aumento de volume na região cervical, disfagia e dispneia transitória. Segundo o proprietário, a massa apareceu e aumentou de tamanho no decorrer de sete meses.

Ao exame clínico, constatou-se aumento de volume, de consistência firme, na posição ventrolateral com discreto deslocamento à esquerda (Figura 1A). No exame radiográfico, visualizou-se uma massa de aspecto oval com conteúdo radiopaco entre C2-C4, característico de divertículo esofágico (Figura 1B), sem a possibilidade de identificação da causa dessa afeç̧ão. O paciente foi então encaminhado para avaliação cirúrgica, momento no qual se optou pela realização de diverticulectomia seguida de esofagorrafia.

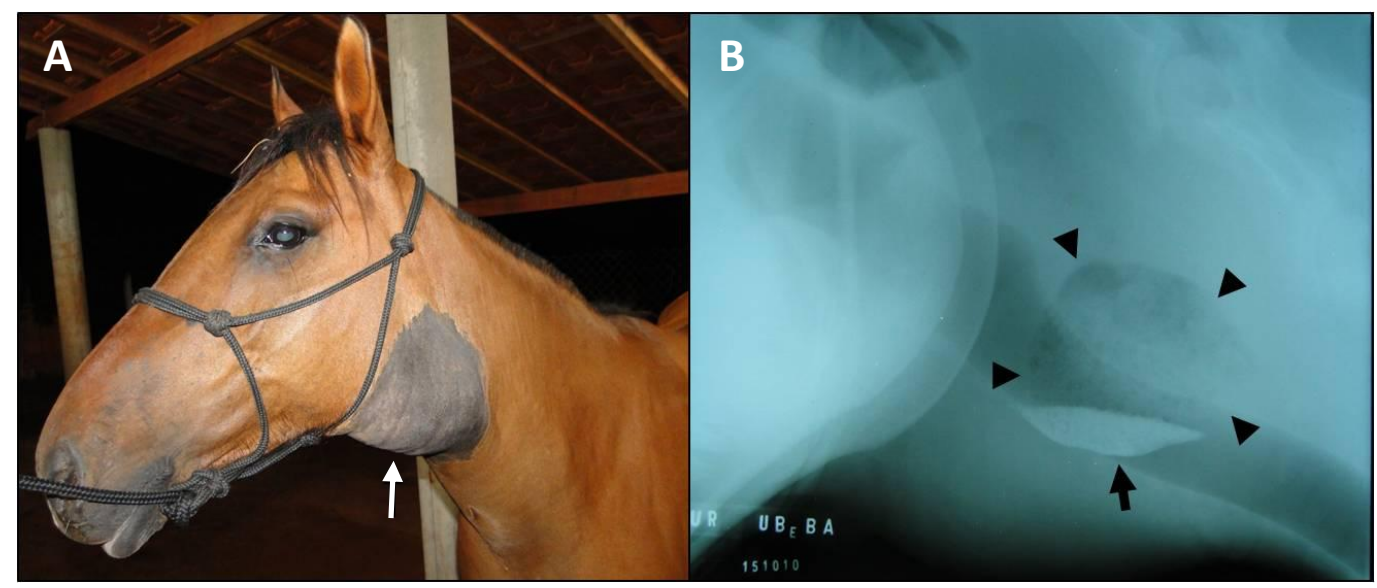

Figura 1 - Equino acometido por divertículo esofágico. Em A, aumento de volume em região cervical ventral (seta). Em B, exame radiográfico indicando o aumento de volume (cabeças de setas) e acúmulo de conteúdo radiopaco (seta) na porção cranial do esôfago. FAFRAM/FE. Ituverava, 2011.

Antes do procedimento cirúrgico, o paciente foi mantido em jejum alimentar por 12 horas e jejum hídrico por seis horas, sendo realizada passagem de sonda nasogástrica antes do procedimento anestésico.

A medicação pré-anestésica utilizada foi xilazina $(0,5 \mathrm{mg} / \mathrm{kg}, \mathrm{IV})$; indução anestésica com quetamina $(2,0 \mathrm{mg} / \mathrm{kg}, \mathrm{IV})$ e diazepan $(0,2 \mathrm{mg} / \mathrm{kg}, \mathrm{IV})$. Na sala de preparo, o paciente foi intubado com sonda orotraqueal número 18 e posicionado em decúbito dorsal sobre a 
mesa, a manutenção anestésica foi feita com isoflurano vaporizado em 100\% de oxigênio em vaporizador universal calibrado. Após tricotomia da região ventral do pescoço, procedeu-se a anti-sepsia prévia que consistiu em lavagem local com solução de iodopovidona degermante seguida de aplicação tópica de álcool 70\%. No centro cirúrgico, foi feita nova anti-sepsia com solução de iodopovidona tópica seguida de aplicação de álcool $70 \%$, em seguida a região cervical foi isolada com panos estéreis.

O procedimento cirúrgico foi iniciado através de uma incisão de pele de aproximadamente $15 \mathrm{~cm}$, realizada na região ventral do pescoço. Após divulsão do subcutâneo, foi possível identificar os pares dos músculos esternotireóideo, esternoióideo e omoióideo. A dissecção desses músculos foi realizada com dificuldade, devido à exuberante aderência dessa musculatura com a massa responsável pelo aumento de volume na região cervical, assim como, pela estreita ligação desta com a artéria carótida esquerda. Isolada a massa, a mesma foi seccionada e identificada como sendo um abscesso. Com auxílio de um bisturi eletrônico ${ }^{1}$, no modo corte e resistência de 200 Ohms, o abscesso foi seccionado gradativamente, até a identificação de uma cápsula muscular que, ao ser incisada, possibilitou a visualização de conteúdo alimentar. Após inspeção minuciosa das estruturas anatômicas, notou-se que a estrutura tratava-se de um divertículo esofágico fistulado envolvido por abscesso (Figura 2).

Foi realizada a diverticulectomia e, em seguida, as camadas esofágicas foram suturadas com fio poliglactina 910 2-0 em padrão Swift. Durante o procedimento, optou-se por deixar um fragmento da cápsula do abscesso que se encontrava aderido à parede da artéria carótida, devido ao risco de rompimento da mesma e de hemorragia severa. O tecido subcutâneo foi suturado com fio poliglactina 910 2-0, em padrão colchoeiro horizontal contínuo, e a dermorrafia realizada com fio nailon 2, em padrão simples separado. No pós-operatório imediato, administrou-se diurético anti-hipertensivo associado a um anti-inflamatório esteroidal (triclormetiazida 0,5 mg/kg e dexametasona 0,025 mg/kg, dose única, IM), antiinflamatório não esteroidal (fenilbutazona, dose de $4,4 \mathrm{mg} / \mathrm{kg}$, IV), e soro antitetânico (25 $\mathrm{UI} / \mathrm{kg}$, dose única, IM).

\footnotetext{
${ }^{1}$ Bisturi Eletrônico BP-100 Plus. Transmai Equipamentos Médicos Hospitalares, São Paulo-SP.
} 


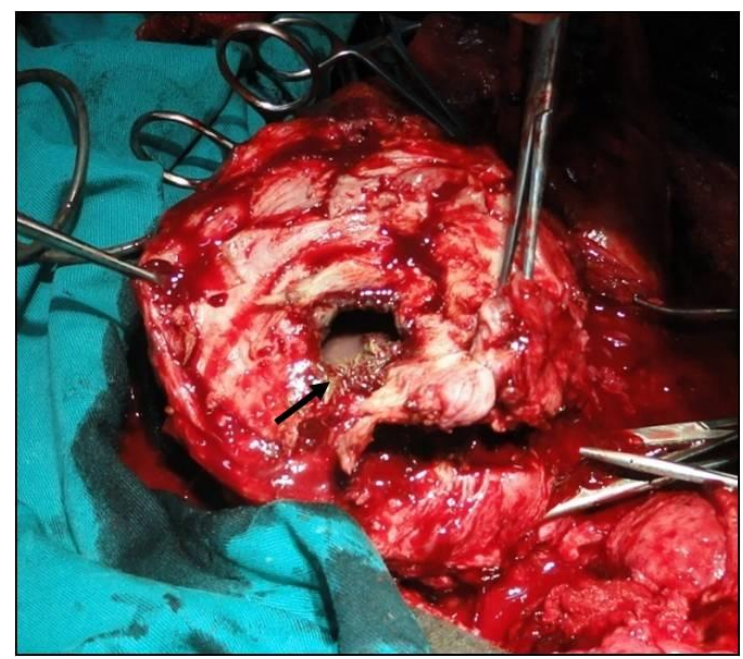

Figura 2 - Divertículo esofágico do equino envolvido por abscesso durante procedimento de diverticulectomia modificada, observar conteúdo alimentar na luz da alteração (seta). FAFRAM/FE. Ituverava, 2011.

Após o procedimento, o paciente recebeu nutrição enteral via sonda nasogástrica e medicação de suporte (Figura 3) baseada na administração de antibióticos (penicilina G procaína $30.000 \mathrm{UI} / \mathrm{kg}$, IM, 12/12 horas, 14 dias; gentamicina, 6,6 mg/kg, IV, 24/24 horas, 14 dias; metronidazol, 15 mg/kg, IV, 12/12 horas, 14 dias), anti-inflamatório não esteroidal (fenilbutazona, 4,4 mg/kg, IV, 24/24 horas, 5 dias) e protetor gástrico de mucosa (omeprazol, $4 \mathrm{mg} / \mathrm{kg}$, via oral, 24/24 horas, 7 dias). Na ferida cirúrgica, optou-se por limpeza com solução fisiológica e solução de iodopovidona tópica, seguida por instilação de rifamicina sódica, 12/12 horas, até a retirada dos pontos.

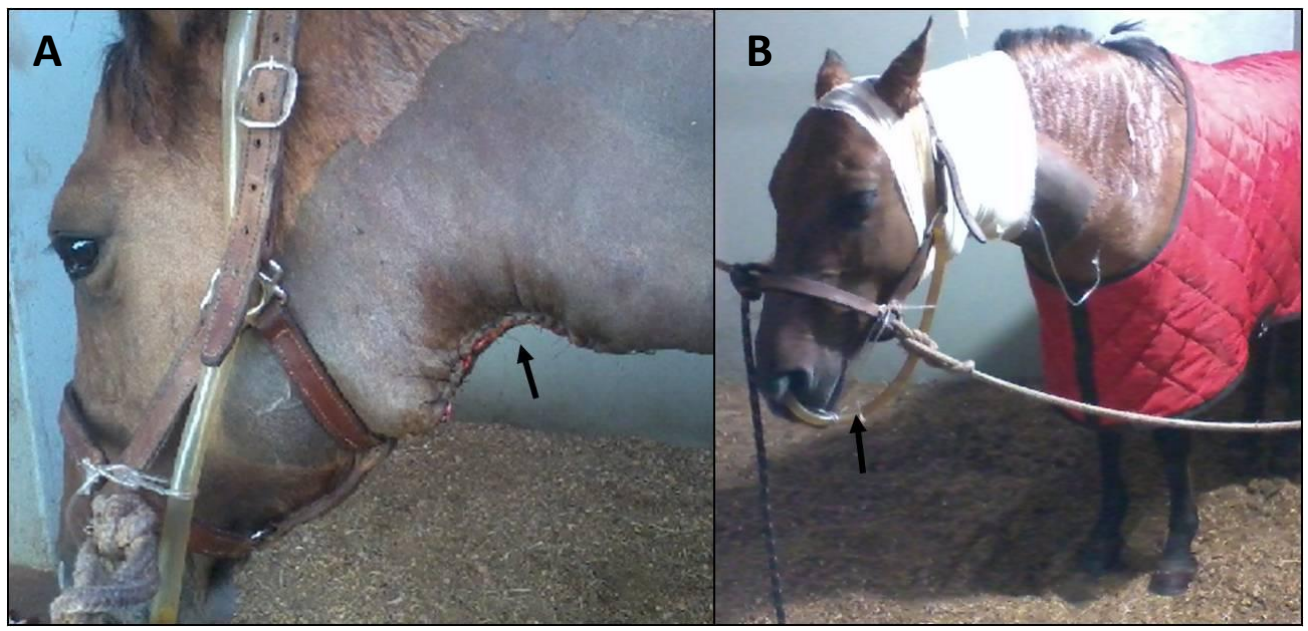

Figura 3 - Equino acometido por divertículo esofágico três dias após o procedimento cirúrgico de diverticulectomia. Em A, aspecto da sutura na região ventral do pescoço do paciente (seta). Em B, paciente sob fluidoterapia e permanência da sonda nasogástrica (seta). FAFRAM/FE. Ituverava, 2011. 
Decorridos três dias do procedimento cirúrgico, mesmo sendo observado edema na região crânio-ventral cervical, próxima a orofaringe, foi oferecida pequena quantidade de feno ao paciente. No entanto, o mesmo sufocou-se e, agitado, atirou-se ao chão; contudo, levantouse rapidamente e sem sinais de sufocamento. O paciente foi contido na baia e, após exame clínico e avaliação da ferida, verificou-se que houve rompimento de alguns dos pontos da sutura da pele e do subcutâneo.

Pressupondo que o rompimento dos pontos proporcionou descompressão da orofaringe e estruturas próximas, aliviando a tensão causada pelo edema e revertendo o sufocamento, optou-se por tratar a ferida por segunda intenção, lavando-a com iodopovidona tópica e aplicando pomada a base de digluconato de clorexidina associada a açúcar cristal até que o tecido de granulação atingisse o nível da pele. Após esse período aplicou-se pomada à base de triancinolona acetonida, sulfato de neomicina, gramicidina e nistatina até a cicatrização completa da mesma (Figura 4A). Não foi oferecido mais alimento para o animal nesse dia.

No dia seguinte à deiscência da sutura, optou-se pelo fornecimento de ração própria para equinos dissolvida em água morna (sopa) com boa aceitabilidade pelo paciente, que se manteve estável, alimentando-se assim durante 20 dias. Após esse período, foi oferecido capim picado associado ao pastejo supervisionado até a alta, quatro meses após o procedimento cirúrgico (Figura 4B).

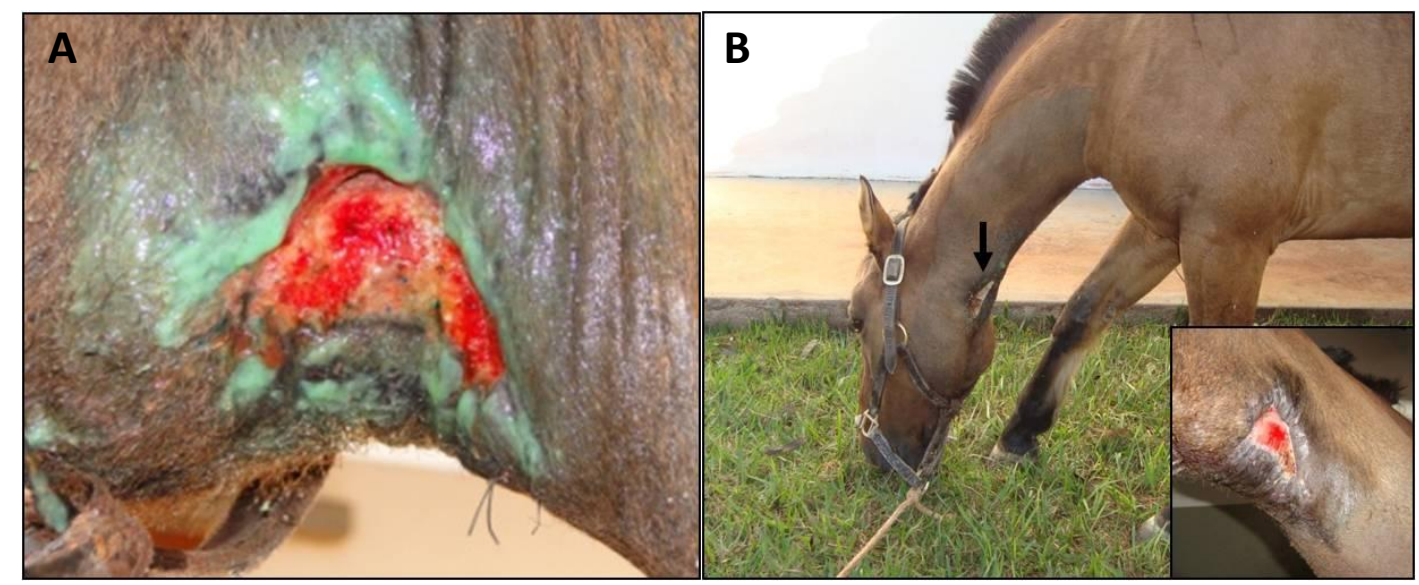

Figura 4 - Equino acometido por divertículo esofágico, oito dias após o procedimento de diverticulectomia. Em $A$, imagem da ferida cirúrgica em tratamento por segunda intenção após o rompimento dos pontos. Em $B$, paciente pastejando após 20 dias do rompimento dos pontos da ferida cirúrgica, no detalhe imagem da ferida cirúrgica em processo de cicatrização. FAFRAM/FE. Ituverava, 2011. 


\section{RESULTADOS E DISCUSSÃO}

O divertículo esofágico é uma afecção bem conhecida na medicina humana (OKANO et al., 2000), no entanto, não são frequentemente descritos na literatura veterinária. Em animais, são encontrados casos relatados em equinos (MACORIS et al., 2008; MURRAY; GAUGHAN, 1993; VRINS et al., 1983), cães (HERNÁNDEZ et al., 2007) e gatos (BÖRKÜ et al., 2009). Portanto, acredita-se que este estudo possa contribuir para o tratamento de equinos que sofram desta alteração.

Conforme informado pelo proprietário na anamnese, sinais como tosse durante a deglutição, ptialismo, regurgitação nasal com conteúdo de saliva e alimentos, são condizentes com obstrução esofágica e consequente divertículo (MURRAY; GAUGHAN, 1993). Stick (1990) descreve que, além do aumento de volume na região cervical que resulta em disfagia, dependendo do grau de obstrução, também pode haver dificuldade durante a passagem de sonda nasogástrica nos animais acometidos.

O esofagograma contrastado é o exame mais indicado para delinear o divertículo e confirmar a afeç̧ão (BLICKSLAGER; JONES, 2006); sendo aquele realizado com contraste a base de bário, útil na diferenciação de um divertículo por pulsão de um por tração (HIGGINS; SNYDER, 2006). No entanto, em casos da afecção estar associada à ocorrência de pneumonia por aspiração, o esofagograma pode não ser fidedigno (MURRAY; GAUGHAN, 1993). Contudo, exames radiográficos simples e endoscópicos também podem ser utilizados no diagnóstico de divertículo esofágico (HIGGINS; SNYDER, 2006; MURRAY, 2000). No presente caso, apenas o exame radiográfico simples foi sugestivo da ocorrência de divertículo esofágico.

O diagnóstico foi confirmado durante o procedimento cirúrgico, que possibilitou, ainda, classificar o divertículo como de tração (ou verdadeiro) devido à presença do abscesso ao redor do mesmo (HIGGINS; SNYDER, 2006); contudo, não foi possível identificar a causa inicial da alteração, como também ocorreu no caso descrito por Murray e Gaughan (1993). A causa inicial de um divertículo esofágico por tração muitas vezes é desconhecida, porém, condições de trauma, incluindo lesões no pescoço e corpos estranhos, geralmente estão associadas à maioria dos casos (AANES, 1976; GREET, 1982). 
Existem algumas técnicas cirúrgicas consolidadas para o tratamento de divertículos esofágicos (MUNÍTIZ et al., 2003; STICK, 1999; STICK, 2011). No entanto, dependendo da localização, da origem do divertículo e das condições clínicas do paciente, torna-se necessária uma análise individual e, possivelmente, uma adaptação da técnica cirúrgica à situação encontrada, para que a correção seja feita de forma satisfatória (MACORIS et al., 2008; MURRAY; GAUGHAN, 1993) e não ocorra morte (BALBINOT et al., 2010) ou indicação de eutanásia (VRINS et al., 1983).

O tratamento mais comumente utilizado é a diverticulectomia, que consiste na redução ou remoção do divertículo esofágico (STICK, 1999; STICK, 2011). No presente caso, a diverticulectomia convencional foi dificultada pela presença do abscesso e por sua aderência em estruturas vitais. A modificação da técnica, seccionando-se a massa gradativamente foi realizada devido à verificação de que a cápsula do abscesso encontrava-se aderida à parede da artéria carótida, necessitando de maiores cuidados na sua dissecação, além do risco de lesão dos nervos glossofaríngeo e vago, responsáveis pela motilidade do órgão (STICK, 2011). No pós-operatório, o sufocamento foi associado à presença de edema no sítio cirúrgico, região que compreende orofaringe, laringe e porção cranial do esôfago, o que dificultou a deglutição e, consequentemente, levou ao episódio que culminou com o rompimento dos pontos. Higgins e Snyder (2006) descrevem que a alimentação no pós-operatório deve ser realizada com uma dieta leve, úmida e pastosa, o que foi então realizado durante 20 dias, proporcionando uma completa recuperação do paciente.

Após a alta, foram feitos contatos esporádicos com o proprietário, para acompanhar a evolução clínica do paciente. Durante esse período não foram relatados indícios de aumento de volume na região cervical nem episódios de disfagia. O paciente manteve uma alimentação normal e, inclusive, encontrava-se gestando no último contato realizado.

\section{CONCLUSÃO}

Concluiu-se, através deste relato de caso, que a ocorrência de um divertículo esofágico fistulado, com formação subsequente de abscesso e aderência a estruturas anatômicas 
nobres, dificulta o tratamento cirúrgico. Dessa forma, faz-se necessária uma análise individual e, dependendo do caso, um procedimento cirúrgico modificado, associado à terapia antibiótica, anti-inflamatória e dietética, adequadas para a sua correção.

\section{FISTULATED ESOPHAGEAL DIVERTICULUM IN A CRIOLLO BREED MARE - CASE REPORT}

\section{ABSTRACT}

$\mathrm{E}$ sophageal diverticula are focal dilatations of the muscular layer of the esophagus and may be congenital or acquired. This study aims to report the case of a Criollo breed mare that showed a firm increase of cervical volume in the ventrolateral position, with a slight left shift, transient dysphagia and dyspnea. Radiographic examination showed a mass of oval appearance with radiopaque content, among C2-C4, characteristic of esophageal diverticulum. During the surgery, there was exuberant adherence of the cervicalmuscles and the left carotid artery with the mass responsible for the increase of volume in the region. The mass was isolated, sectioned and it was found to be an abscess due to a fistulated diverticulum. It was decided to perform a modified diverticulectomy. Through this case, we conclude that the occurrence of a fistulated esophageal diverticulum with subsequent abscess formation and adherence to important structures makes the surgical treatment difficult and, an individual analysis and a modified surgical procedure for its correction are necessary.

Keywords: Abscess. Anatomic change. Dysphagia. Dyspnea. Esophagus.

\section{DIVERTÍCULO ESOFÁGICO FISTULADO EN UN EQUINO DE LA RAZA CRIOLLA - RELATO DE CASO}

\section{RESUMEN}

$\mathrm{D}$ ivertículos esofágicos son dilataciones focales de la capa muscular del esófago y pueden ser congénitas o adquiridas. Este estudio tiene como objetivo informar sobre el caso de una yegua de raza Criolla que mostró un aumento de volumen cervical de consistencia firme, en posición ventrolateral, con una desviación a la izquierda leve, disfagia transitoria y disnea. En el examen radiográfico se visualizó una masa de aspecto ovalado con contenido radiopaco entre $\mathrm{C} 2-\mathrm{C} 4$, característico de divertículo esofágico. Durante la cirugía, se observó exuberante adherencia de la musculatura cervical y la arteria carótida izquierda 
con la masa responsable del aumento en volumen en la región. Aislada la masa, que se seccionó y se encontró que se correspondía con un absceso, secundario a un divertículo, optándose por la realización de una diverticulectomía modificada. A través de este caso, se concluye que la presencia de un divertículo esofágico fistulado con formación posterior de un absceso y adherencia a estructuras importantes, el tratamiento quirúrgico se hace difícil. Un análisis individual y un procedimiento quirúrgico modificado para su corrección, es necesaria.

Palabras clave: Absceso. Alteración anatómico. Disfagia. Disnea. Esófago.

\section{REFERÊNCIAS}

AANES, W. A. The diagnosis and surgical repair of diverticulum of the esophagus.

Proceedings of American Association of Equine Practitioners, v. 21, p. 211-222, 1975.

BALBINOT, M.; SILVA, M. B.; AMORIM, T. M.; et al. Divertículo esofágico em equino. Interbio, v. 4, n. 1, p. 117, 2010.

BLICKSLAGER, A. T.; JONES, S. L. Distúrbios do esôfago. In: SMITH, B. P. Tratado de medicina interna de grandes animais. 3. ed. Barueri: Manole, 2006. 615p.

BÖRKÜ, M. K.; URAL, K.; KIBAR, M.; et al. Esophageal diverticulum in a cat. Turkish Journal of Veterinary and Animal Sciences, v. 33, n. 2, p. 171-174, 2009.

FUBINI, S. L. Esophageal diseases. In: MAIR, T.; DIVERS, T.; DUCHARME, N. Manual of equine gastroenterology. Philadelphia: WB Saunders, 2002. Cap. 7, p. 89-98.

GREET, T. R. C. Observations on the potential role of oesophageal radiography in the horse. Equine Veterinary Journal, v. 14, p. 73-79, 1982.

HERNÁNDEZ, C. A.; GAVIRIA, A.; RESTREPO, R. Divertículo esofágico en un pastor alemán: reporte de caso. Revista Colombiana de Ciencias Pecuarias, v. 20, n. 1, p. 73-78, 2007.

HIGGINS, A. J.; SNYDER, J. R. Esophageal diverticulum. In: HIGGINS, A. J.; SNYDER, J. R. The equine manual. 2. ed. Edinburgh: Elsevier Saunders, 2006, p. 536-537.

MACORIS, D. G.; LOPES, M. C. S.; BERLINGIERI, M. A. Divertículo esofágico em equinos: relato de caso. Revista CFMV, v. 14, n. 44, p. 30-45, 2008.

MUNÍTIZ, V., ORTIZ, A.; HARO, L. F. M.; et al. Diverticulopexia asociada a miotomia del cricofaríngeo en el tratamiento del divertículo de Zenker. Presentación de 21 casos. Cirugía Española, v. 74, n. 5, p. 289-292, 2003. 
MURRAY, R. C.; GAUGHAN, E. M. Pulsion diverticulum of cranial cervical esophagus in a horse. Canadian Veterinary Journal, v. 34. n. 6, p. 365-367, 1993.

MURRAY, M. J. O esôfago. In: REED, S. M.; BAYLY, W. M. Medicina interna equina. Rio de Janeiro: Guanabara Koogan, 2000. Cap. 12, p. 524-529.

OKANO, N.; VARGAS, E. C.; MORIYA, T.; et al. Divertículo do esôfago: análise de 24 pacientes portadores do divertículo de Zenker. Acta Cirurgica Brasileira, v. 15, suppl. 2, p. 60-62, 2000.

STICK, J. A. Esophageal obstruction. In: WHITE, N. A.; MOORE, J. N. Current practice of equine surgery. Philadelphia: Lippincott Williams and Wilkins, 1990, p. 280-296.

STICK, J. A. Diseases of the esophagus. In: COLAHAN, P. T.; MAYHEW, A. M.; MOORE, J. N. Equine medicine and surgery. 5. ed. Missouri: Mosbby, 1999. 677p.

STICK, J. A. Esophagus. In: AUER, J. A.; STICK, J. A. Equine surgery. 4. ed. Philadelphia: Elsevier Saunders, 2011. Cap. 31, p. 367-388.

VRINS, A.; O'BRIEN, T. R.; CARLSON, J. Diverticulum and fistula of the lower cervical esophagus in a horse. Canadian Veterinary Journal, v. 24, n. 12, p. 385-387, 1983. 